Received January 13, 2014 Revised January 22, 2014 Accepted February 4, 2014

Correspondence to:

Ji-Won Ryu

Department of Oral Medicine, School

of Dentistry, Chosun University, 309 ,

Pilmun-daero, Dong-gu, Gwangju

501-759, Korea

Tel: +82-62-220-3897

Fax: +82-62-234-2119

E-mail:dentian@chosun.ac.kr

This study was supported by research fund from Chosun University Dental Hospital, 2013.

\section{Evaluation of Gustatory Function in Patients with Sleep Disordered Breathing}

\author{
Jong-Mo Ahn, Kook-Jin Bae, Chang-Lyuk Yoon, Ji-Won Ryu \\ Department of Oral Medicine, School of Dentistry, Chosun University, Gwangju, Korea
}

Purpose: The aim of this study is to evaluate the difference between gustatory functions in a sleep disordered breathing (SDB) group and a control group. The pathogenesis of SDB has not been fully understood. Though the precise contributions of neuromuscular and anatomical factors on SDB pathogenesis are still debated, we hypothesized that the gustatory dysfunction could be predisposed to SDB.

Methods: All patients were diagnosed as SDB by polysomnography (PSG). On the basis of PSG results, patients were divided into 3 groups: snoring, mixed, and obstructive sleep apnea (OSA). The control group comprised healthy volunteers who were the same age as those of the SDB group and whose breathing was verified as normal using a portable sleep monitor device. The patient group and the control group were evaluated for gustatory functions with an electrogustometry (EGM). The electrical taste thresholds were measured in the anterior, midlateral, and posterior sides of the tongue and soft palatal regions, both sides. To find out the difference in EGM scores, statistical analysis was performed using the Kruskal-wallis and Mann-Whitney U test with 95\% confidence interval and $\mathrm{p}<0.05$ significance level.

Results: The patients with SDB had higher EGM scores than the control group at all spots tested, except for the right midlateral of the tongue, and there was a statistical significance in the comparison between the control group and the divided SDB groups, respectively. Among the divided SDB groups, the snoring group had the most significant differences in the number of the measured spots, but there was no difference among the snoring, mixed, and OSA groups.

Conclusions: These results may suggest that neurologic alterations with sleep disordered breathing could be associated with gustatory dysfunction. In the future, further systemic studies will be needed to confirm this study.

Key Words: Electrogustometry; Gustatory function; Obstructive sleep apnea; Sleep disordered breathing; Snoring

\section{INTRODUCTION}

Sleep disordered breathing (SDB) is a broad term used to describe various distinct or occasionally overlapping respiratory dysfunctions, including primary snoring, obstructive sleep apnea (OSA), central sleep apnea, and hypoventilation. ${ }^{1)}$ The pathogenesis of SDB has not been fully understood. Anatomic factors, such as obesity, maxillomandibular retrognathia, and enlarged tonsils, compromise the size of the upper airway and are well-known risk factors for SDB. In neurologic aspects, long-standing snoring-induced vibrations cause neurogenic lesions in upper airway tissues, thereby damaging the reflex circuits responsible for keeping the upper airway open during inspiration. ${ }^{2)}$ In addition, many studies have suggested that neuromuscular alterations also contribute to the pathogenesis of SDB. ${ }^{3-5)}$ In these studies, several different methods of measuring local sensory neuropathy have been used, such as two-point discrimination, ${ }^{4)}$ vibration, ${ }^{5)}$ and air-pressure pulses ${ }^{5}$; however, there were no attempts to evaluate the gustatory function in the SDB patients. In the studies about SDB, the gustatory dysfunction has been focused on as a side effect after surgical

Copyright (c) 2014 Korean Academy of Orofacial Pain and Oral Medicine. All rights reserved. 
approaches such as uvulopalatopharyngoplasty (UPPP) and radiofrequency tongue base reduction ${ }^{7)}$ to treat SDB. There was a comment related to the taste disturbance after UPPP that there might have been a pre-existing taste disturbance unrelated to the procedure. ${ }^{8)}$ The aim of this study is to evaluate the difference between gustatory functions in a SDB group and a control group.

\section{MATERIALS AND METHODS}

\section{Subjects}

This case-controlled study, comprised 60 patients, all of whom were diagnosed with SDB by polysomnography (PSG) in the Department of Neurology at Chosun University Hospital, from March to December 2010. On the basis of PSG results, these patients were divided into 3 groups: snoring, mixed, and OSA. The control group comprised 44 healthy volunteers whose age was matched that of the SDB group and whose breathing was verified as normal using a portable sleep monitor device (ApneaLink; ResMed, San Diego, CA, USA) offered by Chosun University Dental Hospital. This study was approved by the institutional review board of Chosun University Dental Hospital.

\section{Methods}

The patient group and the control group were evaluated for gustatory functions with an electrogustometry (EGM, EG-IIB; Nagashima Medical Instrument Co., Tokyo, Japan) with a single, flat, circular stainless-steel stimulus probe

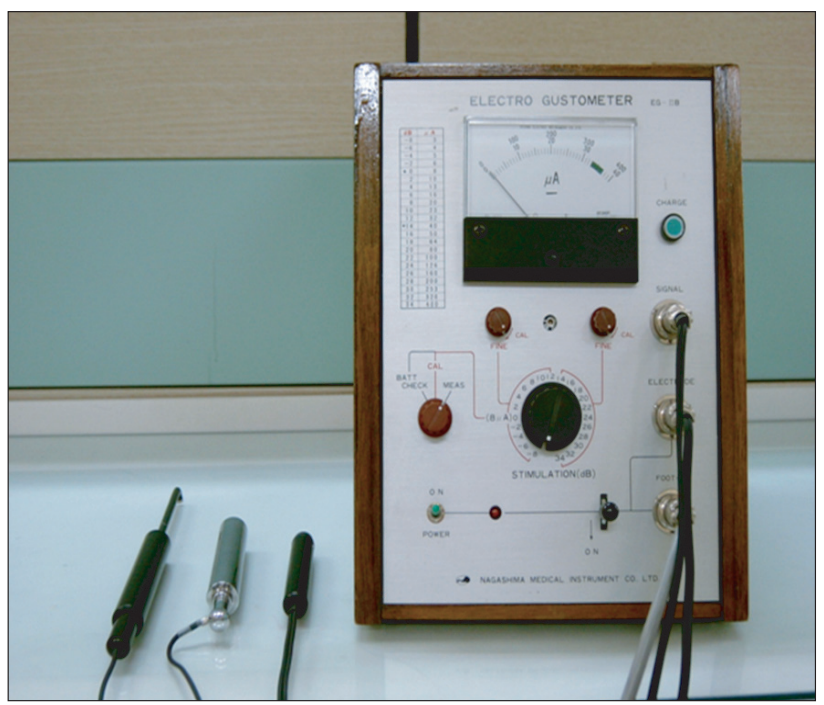

Fig. 1. Electrogustometry (EG- || B; Nagashima Medical Instrument Co.). (5-mm diameter) (Fig. 1). The test was carried out in a comfortable room, free from noise and distraction. Subjects were asked not to eat or drink anything except water 1 hour before testing. The electrical taste thresholds were measured at 8 different sites in the oral cavity: left anterior (LA) 1/3 of the tongue, left midlateral (LM) of the tongue, left posterior (LP) $1 / 3$ of the tongue (circumvallate papillae), left soft (LS) palate, and right anterior (RA) 1/3 of the tongue, right midlateral (RM) of the tongue, right posterior (RP) $1 / 3$ of the tongue (circumvallate papillae), right soft (RS) palate (Fig. 2). After gargling with $5 \mathrm{~mL}$ of distilled water for approximately 10 seconds, the subject rested for 3 minutes. Then, the negative electrode was applied to the subject's right hand, the buzzer to the left hand, and the positive electrode to the recording sites. An electrical stimuli of 1 second was given to the patients, with the current intensity initiated at $-8 \mathrm{~dB}$ and subsequently increased by $2 \mathrm{~dB}$ each time until a taste was evoked. The taste threshold was defined as the lowest detected level of sour, bitter, or metallic taste. The electrical taste thresholds of subjects who did not respond at $34 \mathrm{~dB}$ were regarded as $34 \mathrm{~dB}$.

\section{Statistical Analysis}

All statistical analyses were performed using IBM SPSS Statistics 20.0 (IBM Co., Armonk, NY, USA). To find out the difference in EGM scores, statistical analysis was performed

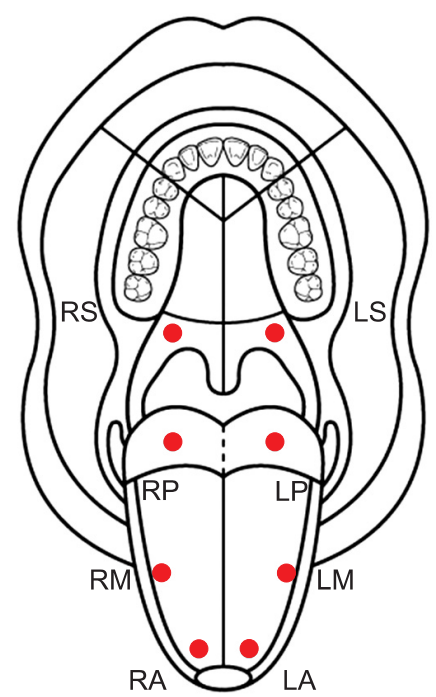

Fig. 2. Measured sites in oral cavity by electrogustometery. RS, right soft palate; LS, left soft palate; RP, right posterior $1 / 3$ of the tongue; $L P$, left posterior $1 / 3$ of the tongue; $R M$, right middle $1 / 3$ of the tongue; LM, left middle $1 / 3$ of the tongue; RA, right tongue tip; LA, left tongue tip. 
using the Kruskal-Wallis and Mann-Whitney U test. Statistical significance was defined as $\mathrm{p}<0.05$, with a $95 \%$ confidence interval.

\section{RESULTS}

There were 19 patients in the snoring group, 22 patients in the mixed group, and 19 patients in the OSA group. The average patient ages and the male : female ratio in each group are listed in Table 1.

In the first analysis, comparison between the control group and all SDB patients, patients with SDB had higher EGM scores than the control group at all spots tested, except for the RM $(p<0.05)$ (Table 2). In the comparison between the control groups and divided SDB groups using the

Table 1. Comparison of age and sex ratio among groups

\begin{tabular}{lcccc}
\hline & Control & \multicolumn{4}{c}{ Patient $(n=60)$} \\
\cline { 3 - 5 } & $(n=44)$ & Snoring $(n=19)$ & Mixed $(n=22)$ & OSA $(n=19)$ \\
\hline Age (y) & $42.0 \pm 4.6$ & $44.73 \pm 10.5$ & $44.6 \pm 11.5$ & $46.7 \pm 11.3$ \\
sex (M : F) & $40: 4$ & $16: 3$ & $20: 2$ & $14: 5$ \\
\hline
\end{tabular}

OSA, obstructive sleep apnea; M, male; F, female.

Values are presented as mean \pm standard deviation.
Kruskal-Wallis test, there was a statistically significant difference in the five tested sites (LA, RP, LP, RS, LS) (Table 3), and there was a statistical significance in the comparison between the control group and divided SDB groups, respectively $(\mathrm{p}<0.05)$. Among the divided SDB groups, the snoring group had the most significant differences in the number of the measured spots, but there was no difference among the snoring, mixed, and OSA groups ( $p>0.05$ ) (Table 4).

\section{DISCUSSION}

SDB is a common sleep disorder that describes various distinct or occasionally overlapping syndromes related to respiratory dysfunction during sleep, from primary snoring to OSA. $^{\text {9) }}$ Especially OSA is a common, chronic disorder that is characterized by sleep fragmentation due to apnea, hypopnea, and repeated arousals resulting from partial or complete closure of the upper airway, and occurs in patients of all ages. ${ }^{10)}$ Anatomic factors such as a narrow upper airway may predispose a patient to SDB, ${ }^{11)}$ although having a narrow airway does not guarantee that a subject will have this condition. It is possible that the narrowed anatomical size and the blunted neuromuscular responses

Table 2. Comparison of electrogustometry scores between control and sleep disordered breathing group (unit: $\mathrm{dB}$ )

\begin{tabular}{lcccccccc}
\hline & \multicolumn{8}{c}{ Location } \\
\cline { 2 - 8 } & LA & RA & LM & RM & LP & RP & LS & RS \\
\hline Control $(n=44)$ & $5.68 \pm 7.40$ & $4.73 \pm 7.60$ & $13.64 \pm 8.98$ & $14.05 \pm 9.66$ & $12.55 \pm 7.97$ & $12.23 \pm 7.93$ & $9.32 \pm 7.57$ & $11.50 \pm 8.70$ \\
SDB ( $n=60)$ & $11.50 \pm 11.03$ & $9.70 \pm 11.81$ & $18.02 \pm 11.45$ & $17.13 \pm 11.61$ & $20.10 \pm 11.72$ & $18.30 \pm 12.02$ & $19.80 \pm 12.97$ & $17.87 \pm 11.44$ \\
$\mathrm{p}$-value & $0.010^{*}$ & $0.035^{*}$ & $0.041^{*}$ & 0.119 & $0.001^{*}$ & $0.008^{*}$ & $0.000^{*}$ & $0.004^{*}$ \\
\hline
\end{tabular}

$L A$, left tongue tip; $R A$, right tongue tip; $L M$, left middle $1 / 3$ of the tongue; $R M$, right middle $1 / 3$ of the tongue; $L P$, left posterior $1 / 3$ of the tongue; RP, right posterior $1 / 3$ of the tongue; LS, left soft palate; RS, right soft palate; SDB, sleep disordered breathing.

Values are presented as mean \pm standard deviation.

${ }^{*} \mathrm{p}<0.05$ (by Mann-Whitney U test).

Table 3. Comparison of electrogustometry scores between control and devided sleep disordered breathing group (unit : $\mathrm{dB}$ )

\begin{tabular}{lcccccccc}
\hline & \multicolumn{8}{c}{ Location } \\
\cline { 2 - 9 } & \multicolumn{1}{c}{ LA } & RA & LM & \multicolumn{1}{c}{ RM } & LP & RP & LS \\
\hline Control & $5.68 \pm 7.40$ & $4.73 \pm 7.60$ & $13.64 \pm 8.98$ & $14.05 \pm 9.66$ & $12.55 \pm 7.97$ & $12.23 \pm 7.93$ & $9.32 \pm 7.57$ & $11.50 \pm 8.70$ \\
Snoring & $10.63 \pm 8.85$ & $9.89 \pm 10.21$ & $16.84 \pm 9.92$ & $20.00 \pm 10.52$ & $21.16 \pm 11.34$ & $19.58 \pm 12.08$ & $19.80 \pm 12.97$ & $17.87 \pm 11.44$ \\
Mixed & $13.00 \pm 11.00$ & $8.73 \pm 11.81$ & $19.05 \pm 12.38$ & $15.18 \pm 12.20$ & $19.63 \pm 12.24$ & $19.73 \pm 13.46$ & $18.82 \pm 14.85$ & $16.10 \pm 12.27$ \\
OSA & $10.63 \pm 8.85$ & $9.89 \pm 10.21$ & $16.84 \pm 9.92$ & $20.00 \pm 10.52$ & $21.16 \pm 11.34$ & $19.58 \pm 12.08$ & $20.53 \pm 12.34$ & $18.95 \pm 10.59$ \\
p-value & $0.042^{*}$ & 0.192 & 0.220 & 0.187 & $0.010^{*}$ & $0.049 *$ & $0.000^{*}$ & $0.023^{*}$ \\
\hline
\end{tabular}

$L A$, left tongue tip; $R A$, right tongue tip; $L M$, left middle $1 / 3$ of the tongue; $R M$, right middle $1 / 3$ of the tongue; $L P$, left posterior $1 / 3$ of the tongue; RP, right posterior $1 / 3$ of the tongue; LS, left soft palate; RS, right soft palate; OSA, obstructive sleep apnea.

Values are presented as mean \pm standard deviation.

${ }^{*} p<0.05$ (by Kruskal-Wallis test). 
Table 4. Comparison of $\mathrm{p}$-values between control and each group of sleep disordered breathing

\begin{tabular}{lcccccccc}
\hline & \multicolumn{8}{c}{ Location } \\
\cline { 2 - 9 } & LA & RA & LM & RM & LP & RP & LS & RS \\
\hline Control vs. snoring & $0.049^{*}$ & 0.050 & 0.169 & $0.026^{*}$ & $0.006^{*}$ & $0.018^{*}$ & $0.000^{*}$ & $0.006^{*}$ \\
Control vs. mixed & $0.008^{*}$ & 0.231 & 0.079 & 0.692 & $0.019^{*}$ & $0.043^{*}$ & $0.008^{*}$ & 0.172 \\
Control vs. OSA & 0.347 & 0.126 & 0.181 & 0.375 & $0.024^{*}$ & 0.153 & $0.001^{*}$ & $0.021^{*}$ \\
\hline
\end{tabular}

$L A$, left tongue tip; $R A$, right tongue tip; $L M$, left middle $1 / 3$ of the tongue; $R M$, right middle $1 / 3$ of the tongue; $L P$, left posterior $1 / 3$ of the tongue; RP, right posterior 1/3 of the tongue; LS, left soft palate; RS, right soft palate; OSA, obstructive sleep apnea.

${ }^{*} \mathrm{p}<0.05$ (Mann-Whitney $U$ test).

of upper airways are both required for the development of SDB. ${ }^{12)}$ Many studies investigated the relationship between a peripheral neuropathy of the upper airway and SDB. The mechanoreceptors that respond to changes in airway pressure, airflow, and temperature, especially those involved with afferent sensory receptors, could indirectly play a role in maintaining upper airway patency. ${ }^{10)}$ Gustatory function is mediated by special sensory receptor neurons. These sensory receptors are innervated by the chorda tympani and greater superficial petrosal nerves from geniculate ganglia in the anterior oral cavity, by the glossopharyngeal nerves from petrosal ganglia in the posterior oral cavity, and by the superior laryngeal nerves from nodose ganglia in the epiglottis. ${ }^{13)}$ Although there are different nerve innervations between afferent sensory receptors and special sensory taste receptors, the long-standing mechanical vibrations could cause neurogenic lesions in upper airway tissues, ${ }^{2)}$ so it could be applied to taste buds where taste receptors are located. Therefore we hypothesized that gustatory dysfunction could be predisposed to SDB. To the best of our knowledge, gustatory function in SDB patients has not been evaluated yet.

We evaluate gustatory function using EGM. Chemosensory-based gustatory testing (the 3-drop method, impregnated taste strips, spatial taste test) is useful in the research setting for testing taste, but it is time-consuming and complicated. ${ }^{7)}$ In contrast, EGM is a quick, repeatable, and quantifiable method of assessing taste dysfunction. ${ }^{14)}$ Its reliability and validity have been evaluated in previous clinical studies. ${ }^{15,16)}$ The results of this study showed that SDB patients had statistically significant higher EGM scores than the control group in all area except for right middle (RM) (Table 2). In previous studies, evaluating gustatory function, the difference between groups was assessed using independent t-tests. ${ }^{7)}$ Although independent t-tests are often used to determine the differences between groups, we analyzed the data with the Mann-Whitney U test and Kruskal-Wallis test, characterized as non-parametric statistics. These tests were used because the distribution of the obtained data did not meet normal distribution conditions, and the data were considered as orderly variables. We also evaluated the difference between groups using the independent t-tests, and the results were the same.

In the comparison between the control groups and divided SDB groups using the Kruskal-Wallis test, there was a statistically significant difference in the five tested sites (LA, RP, LP, RS, LS) (Table 3), and there was a statistical significance in the comparison between the control group and divided SDB groups, respectively ( $<<0.05)$. Notably, among the three SDB groups, the snoring group had the most significant differences in the number of the measured spots (Table 4). These results suggest that sustained mechanical vibration could be associated with neurologic alteration in gustatory function, although there was no difference among the snoring, mixed, and OSA groups ( $>>0.05)$. Generally, OSA seems to be progressive over time, and many patients reported years of snoring before witnessing apneas and symptoms. ${ }^{2,17)}$ So we hypothesized that the electrical taste thresholds might increase over the stages of SBD, from primary snoring to OSA. However, the results did not match our expectations, possibly due to the manner in which the SDB patients were divided. Although the results might be clinically significant, further studies are indicated to evaluate the progression of SDB quantitatively.

This study has several limitations. First, we did not use a questionnaire to assess subjective symptoms of tastes. It should be needed to correlate the subjective symptoms and objective signs. A validated questionnaire or chemosensory-based gustatory testing should be combined with EGM in future studies. Second, we divided into patient group, 
according to the PSG results, but the results of apneahypopnea index and snoring index were not considered quantitatively in this study. In the future, analyses correlating the progression of SDB and gustatory function will be needed to further evaluate the results of this study.

\section{CONFLICT OF INTEREST}

No potential conflict of interest relevant to this article was reported.

\section{REFERENCES}

1. Panossian L, Daley J. Sleep-disordered breathing. Continuum (Minneap Minn) 2013;19:86-103.

2. Sunnergren 0, Broström A, Svanborg E. Soft palate sensory neuropathy in the pathogenesis of obstructive sleep apnea. Laryngoscope 2011;121:451-456.

3. Kimoff RJ, Sforza E, Champagne V, Ofiara L, Gendron D. Upper airway sensation in snoring and obstructive sleep apnea. Am J Respir Crit Care Med 2001;164:250-255.

4. Guilleminault C, Li K, Chen NH, Poyares D. Two-point palatal discrimination in patients with upper airway resistance syndrome, obstructive sleep apnea syndrome, and normal control subjects. Chest 2002;122:866-870.

5. Nguyen AT, Jobin V, Payne R, Beauregard J, Naor N, Kimoff RJ. Laryngeal and velopharyngeal sensory impairment in obstructive sleep apnea. Sleep 2005;28:585-593.

6. Li HY, Lee LA, Wang PC, et al. Taste disturbance after uvulopalatopharyngoplasty for obstructive sleep apnea. Otolaryngol Head
Neck Surg 2006;134:985-990.

7. Eun YG, Shin SY, Byun JY, Kim MG, Lee KH, Kim SW. Gustatory function after radiofrequency tongue base reduction in patients with obstructive sleep apnea. Otolaryngol Head Neck Surg 2011;145:853-857.

8. Enoz M. Was there evidence of pre-existing taste disturbance in the five patients with postoperative abnormality? Otolaryngol Head Neck Surg 2007;137:176, author reply 176.

9. Flemons WW. Clinical practice. Obstructive sleep apnea. N Engl J Med 2002;347:498-504.

10. Tsai YJ, Ramar K, Liang YJ, et al. Peripheral neuropathology of the upper airway in obstructive sleep apnea syndrome. Sleep Med Rev 2013;17:161-168.

11. Jung JK, Hur YK, Choi JK. The effect of mandibular protrusion on dynamic changes in oropharyngeal caliber. Korean J Oral Med 2010;35:193-202.

12. Patil SP, Schneider H, Marx JJ, Gladmon E, Schwartz AR, Smith PL. Neuromechanical control of upper airway patency during sleep. J Appl Physiol (1985) 2007;102:547-556.

13. Matsumoto I. Gustatory neural pathways revealed by genetic tracing from taste receptor cells. Biosci Biotechnol Biochem 2013;77:1359-1362.

14. Tomita H, Ikeda M. Clinical use of electrogustometry: strengths and limitations. Acta Otolaryngol Suppl 2002;(546):27-38.

15. Stillman JA, Morton RP, Hay KD, Ahmad Z, Goldsmith D. Electrogustometry: strengths, weaknesses, and clinical evidence of stimulus boundaries. Clin Otolaryngol Allied Sci 2003;28:406410.

16. Lobb B, Elliffe DM, Stillman JA. Reliability of electrogustometry for the estimation of taste thresholds. Clin Otolaryngol Allied Sci 2000;25:531-534.

17. Lugaresi E, Plazzi G. Heavy snorer disease: from snoring to the sleep apnea syndrome--an overview. Respiration 1997;64(Suppl 1):11-14. 